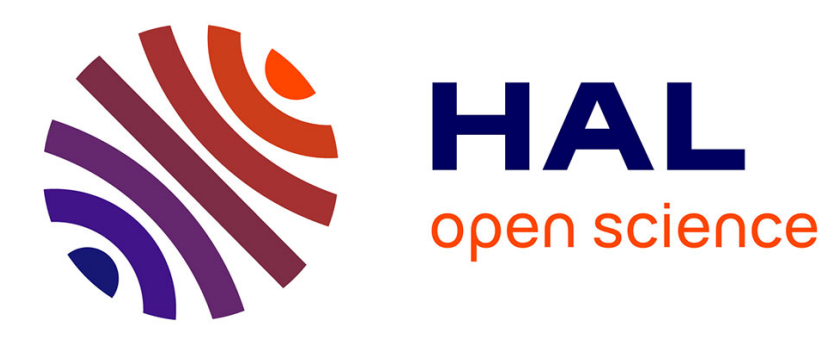

\title{
Surface plasmon polaritons on metal cylinders with dielectric core
}

\author{
U. Schröter, Alain Dereux
}

\section{To cite this version:}

U. Schröter, Alain Dereux. Surface plasmon polaritons on metal cylinders with dielectric core. Physical Review B: Condensed Matter and Materials Physics (1998-2015), 2001, 64 (12), pp.125420. hal00473024

\section{HAL Id: hal-00473024 \\ https://hal.science/hal-00473024}

Submitted on 13 Apr 2010

HAL is a multi-disciplinary open access archive for the deposit and dissemination of scientific research documents, whether they are published or not. The documents may come from teaching and research institutions in France or abroad, or from public or private research centers.
L'archive ouverte pluridisciplinaire HAL, est destinée au dépôt et à la diffusion de documents scientifiques de niveau recherche, publiés ou non, émanant des établissements d'enseignement et de recherche français ou étrangers, des laboratoires publics ou privés. 


\title{
Surface plasmon polaritons on metal cylinders with dielectric core
}

\author{
Ursula Schröter and Alain Dereux \\ Laboratoire de Physique, Optique Submicronique, Université de Bourgogne, 9 avenue Alain \\ Savary, F-21078 Dijon, France
}

(April 9, 2001)

\begin{abstract}
Metal cladded dielectric cylinders with submicron diameters may serve to model coated tips used in near-field scanning optical microscopy. The signal measured may be greatly influenced by resonance effects due to eigenmodes of the probe. Especially, using a photon scanning tunneling microscope setup, gold coated tips have been found to detect a signal proportional to the magnetic field distributions [E.Devaux et al., Phys. Rev. B 62, 10504 (2000)]. This effect is attributed to cylindrical surface plasmons. We present here fully retarded calculations of the dispersion and field patterns of the non-radiative plasmon modes in cylindrical geometry. We study the effect of varying the cylinder radius on the surface plasmon dispersion, thus justifying that the cylinder is a useful model for near field probes in spite of their slightly conical shape.
\end{abstract}

73.20.Mf,41.20.-q,78.20.-e,78.20.Bh 


\section{INTRODUCTION}

Surface plasmon polaritons ${ }^{1}$ are electromagnetic eigenmodes of metal-dielectric interfaces. They are characterized by high field enhancements located close to the surfaces. Gold and silver are especially favourable for surface plasmon excitations in the visible range of frequencies. Multilayer structures can support several of these modes ${ }^{2}$. As evanescent waves, surface plasmons persist even when layer thicknesses become so small that waveguide modes cannot be sustained any more.

In scanning near-field optical microscopy (SNOM) pulled dielectric fibres, with or without metal cladding, are used as probes which illuminate the sample locally. Making the tip dimensions much smaller than the wavelength of the light leads to sources of light which, under some specific circumstances, can be close to the ideal definition of a point-like source. However, this is usually paid for by bad throughput properties of the probe and thus by low signal intensity. Plasmon resonances are therefore of great interest for signal amplification and have already been exploited in different SNOM configurations ${ }^{3,4}$.

On the other hand, the properties of the probe, such as eigenmodes sustained by it, may largely influence the nature of the obtained signal. The quantity measured in photon scanning tunneling microscopy (PSTM), where the tip does not illuminate the sample but collect the light close to the sample, is usually related to the local intensity of the electric field. Devaux and co-workers ${ }^{5,6}$ recently showed that gold coated tips can measure a signal proportional to the magnetic field if their dimensions are carefully chosen such that the circular symmetry plasmon modes can be excited at the frequency of the incident light.

In this article we improve on the calculation of the dispersion of the cylindrical plasmons presented in references 5 and 6 by taking into account retardation. In order to estimate the influence of the conical shape of a PSTM tip, we study the dependence of the plasmon dispersion on the diameter of the cylinder. We explicitely calculate the distributions of the electric and the magnetic field with the aim of understanding possible coupling mechanisms.

\section{DISPERSION RELATIONS}

The system under consideration is a metal cylinder with a dielectric core characterized by $\epsilon_{c}$ embedded in an outside medium of infinite extent described by $\epsilon_{a}$ (see inset Fig.2a). These dielectric functions, $\epsilon_{c}=2$ (glass) and $\epsilon_{a}=1$ (air) are taken to be constant throughout this article, although a frequency dependence would present no further difficulty. The metal is also described by a dielectric function $\epsilon(\omega)$, so no charge or current appears explicitely in Maxwell's equations. Data for gold and silver have been taken from Ref.7; the imaginary part of $\epsilon(\omega)$ has been ignored when describing the eigenmodes. Solving Maxwell's equations in cylindrical coordinates $(r, \theta, z)$ leads to the following expressions for the electric and magnetic fields ${ }^{8}$ :

$$
\begin{aligned}
& E_{r}=\left(\frac{i k_{z}}{k_{j}} a_{n}^{j} f_{n}^{j^{\prime}}\left(k_{j} r\right)-\frac{\omega n}{k_{j}^{2} r} b_{n}^{j} f_{n}^{j}\left(k_{j} r\right)\right) S_{n} \\
& E_{\theta}=\left(-\frac{n k_{z}}{k_{j}^{2} r} a_{n}^{j} f_{n}^{j}\left(k_{j} r\right)-\frac{i \omega}{k_{j}} b_{n}^{j} f_{n}^{j^{\prime}}\left(k_{j} r\right)\right) S_{n}
\end{aligned}
$$




$$
\begin{aligned}
& E_{z}=a_{n}^{j} f_{n}^{j}\left(k_{j} r\right) S_{n} \\
& H_{r}=\left(\frac{n \epsilon_{j} \omega}{k_{j}^{2} r} a_{n}^{j} f_{n}^{j}\left(k_{j} r\right)+\frac{i k_{z}}{k_{j}} b_{n}^{j} f_{n}^{j^{\prime}}\left(k_{j} r\right)\right) S_{n} \\
& H_{\theta}=\left(\frac{i \epsilon_{j} \omega}{k_{j}} a_{n}^{j} f_{n}^{j^{\prime}}\left(k_{j} r\right)-\frac{n k_{z}}{k_{j}^{2} r} b_{n}^{j} f_{n}^{j}\left(k_{j} r\right)\right) S_{n} \\
& H_{z}=b_{n}^{j} f_{n}^{j}\left(k_{j} r\right) S_{n}
\end{aligned}
$$

where $i$ is the imaginary unit, $a_{n}^{j}, b_{n}^{j}$ are complex coefficients and $n$ is an integer $(n=$ $0,1,2, \ldots) . k_{z}$ is the wave vector component in $z$-direction (along the cylinder axis), which will be real and the same for all media. $\epsilon_{j}$ is the dielectric function of the medium $j$ and may depend on the frequency $\omega$. The index $j$ can be $a=$ air, $m=$ metal or $c=$ core. As we are not dealing with magnetic materials, the magnetic permeability $\mu$ is considered to be unity everywhere and has been omitted in the equations. $S_{n}$ is the exponential factor

$$
S_{n}=\exp \left(i n \theta+i k_{z} z-i \omega t\right)
$$

and $k_{j}$ is the radial component of the wave vector in the $j$ th medium:

$$
k_{j}^{2}=\epsilon_{j}(\omega / c)^{2}-k_{z}^{2}
$$

where $c$ is the speed of light in vacuum.

Of course, a general solution is a superposition of waves of all $n$, but an eigenmode of the cylinder will be characterized by a fixed $n$ which has to be the same for all media. We only consider non-radiative modes. Therefore in the outermost medium (air) $k_{a}$ is imaginary and $f_{n}^{a}\left(k_{a} r\right)$ is the modified Bessel function $K_{n}\left(k_{a} r\right)$. In the metal, $\epsilon_{m}$ is negative for frequencies in the visible and $k_{m}$ is also imaginary; in this finite region not containing the $z$-axis (that is $r=0) f_{n}^{m}\left(k_{m} r\right)$ has to be taken as a linear combination of the modified Bessel functions $I_{n}\left(k_{m} r\right)$ and $K_{n}\left(k_{m} r\right)$. In the dielectric core, $f_{n}^{c}\left(k_{c} r\right)$ is the modified Bessel function $I_{n}\left(k_{c} r\right)$ for $k_{c}$ imaginary and the ordinary Bessel function $J_{n}\left(k_{c} r\right)$ for $k_{c}$ real; because the core region is finite, a confined cylinder mode may be propagative in radial direction there. In the modified Bessel functions, arguments are understood to be taken without the imaginary unit $i$. In every case $f_{n}^{j^{\prime}}(x)$ denotes the derivative of $f_{n}^{j}(x)$ with respect to its argument $x$.

Applying the boundary conditions that the tangential components of $\vec{E}$ and $\vec{H}$ must be continuous at the inner and outer cylinder radius leads to the sought dispersion relation $\omega\left(k_{z}\right)$. Matching the field components at the boundaries results in a system of linear equations for the coefficients $a_{n}^{j}$ and $b_{n}^{j}$. (In the metal there are four coefficients, instead of only two for the core as well as for the outside region, because $f_{n}$ is a superposition of $I_{n}$ and $K_{n}$ there.) A non-trivial solution of the coefficients exists, if the determinant of this system of linear equations vanishes. The dispersion of possible eigenmodes of the cylinder is obtained by finding numerically the zeros of this determinant on a grid $\left(k_{z}, \omega\right)$. This is a general procedure and the result includes all electromagnetic eigenmodes of the cylinder ${ }^{9}$, surface plasmons as well as waveguide modes. However, in the frequency range considered here, waveguide modes only appear for cylinder radii larger than the examples shown in this article.

For comparison, Fig.1 shows the even simpler case of a full metal cylinder embedded in a dielectric; here, $f_{n}^{m}\left(k_{m} r\right)$ has to be taken as $I_{n}\left(k_{m} r\right)$. Non-radiative plasmon modes 
exist to the right of the light line of the surrounding dielectric (air). The $n=0$ mode goes down to $\omega=0$ approaching the light line from the right. The $n=1$ mode also shows strong retardation effects, but intersects the light line at finite $\omega$ (we only calculated the dispersion in the non-radiative region). The modes of different $n$ rest well separated, there is not any crossing. And even if the curves get broadened by damping, a significant overlap is not expected between branches for at least up to $n=3$.

For the metal cylinder with dielectric core (Fig.2), non-radiative eigenmodes also exist to the right of the light line of the embedding medium (air). However, up to the glass light line the radial part of the wave vector is real in the core. The radial part of the functions given for the field components implies the normal Bessel function to the left and the modified Bessel function to the right of the glass light line. As to the dispersion of the eigenmodes, the transition between these two regions is nevertheless continuous. On the glass light line $k_{z}^{2}=\epsilon_{c}(\omega / c)^{2}$ and $k_{j}=k_{c}$ vanishes in the core medium. Eventually ambiguous expressions with $k_{j}$ appearing in the denominator as in eqs.(1) can be avoided, as the solution for the field components in this case is given in the following closed form:

$$
\begin{aligned}
& E_{r}=\left(\beta_{n}^{c} r^{n-1}-\frac{i n \alpha_{n}^{c}}{2}\left(\frac{r^{n-1}}{k_{z}}+\frac{k_{z} r^{n+1}}{n+1}\right)\right) S_{n} \\
& E_{\theta}=\left(i \beta_{n}^{c} r^{n-1}+\frac{n \alpha_{n}^{c}}{2}\left(\frac{r^{n-1}}{k_{z}}-\frac{k_{z} r^{n+1}}{n+1}\right)\right) S_{n} \\
& E_{z}=\alpha_{n}^{c} r^{n} S_{n} \\
& H_{r}=\left(-\frac{i k_{z}}{\omega} \beta_{n}^{c} r^{n-1}+\frac{k_{z} n \alpha_{n}^{c}}{2 \omega}\left(\frac{r^{n-1}}{k_{z}}+\frac{k_{z} r^{n+1}}{n+1}\right)\right) S_{n} \\
& H_{\theta}=\left(\frac{k_{z}}{\omega} \beta_{n}^{c} r^{n-1}+\frac{i k_{z} n \alpha_{n}^{c}}{2 \omega}\left(\frac{r^{n-1}}{k_{z}}-\frac{k_{z} r^{n+1}}{n+1}\right)\right) S_{n} \\
& H_{z}=\frac{i k_{z}}{\omega} \alpha_{n}^{c} r^{n} S_{n}
\end{aligned}
$$

for $n>0$ and

$$
\begin{gathered}
E_{r}=-i \alpha_{0}^{c} \frac{k_{z} r}{2} S_{0}, \quad E_{\theta}=0, \quad E_{z}=\alpha_{0}^{c} S_{0} \\
H_{r}=0, \quad H_{\theta}=-i \alpha_{0}^{c} \frac{\epsilon_{c} \omega r}{2} S_{0}, \quad H_{z}=0
\end{gathered}
$$

for $n=0 . \alpha_{n}^{c}$ and $\beta_{n}^{c}$ are complex coefficients replacing $a_{n}^{c}$ and $b_{n}^{c}$ in this case. No boundary conditions have been applied yet: (4) is the general solution of Maxwell's equations in a homogeneous medium in cylindrical coordinates for the special case $k_{z}^{2}=\epsilon(\omega / c)^{2}$. The solution has however already been restricted to the type being finite at $r=0$ as we only apply it to the core of the cylinder.

For the metal cylinder with dielectric core there are two surface plasmon branches for each $n$. The lower mode corresponds to a symmetric polarization charge distribution on the inner and the outer surface of the metal cylinder and the upper mode to an asymmetric one. As for the full cylinder the $n=0$ and the $n=1$ mode show strong dispersion whereas the higher order modes are nearly dispersionless. Relatively to the case of the full cylinder, the $n=0$ and the $n=1$ lower branches do not only come much closer together, they even cross. Keeping the core radius fixed at $b=80 \mathrm{~nm}$, in Fig.2 we show the dispersion for the 
two thicknesses $d=32 \mathrm{~nm}$ and $d=24 \mathrm{~nm}$ of the gold cladding corresponding to the ratios $a / b=1.4$ and $a / b=1.3$. Indeed, tips of these dimensions have been reported in references 5 and 6 to provide measurements proportional to the optical magnetic field when using a green laser $(\lambda=543 \mathrm{~nm})$ in the first and when using a red laser $(\lambda=633 \mathrm{~nm})$ in the second case. These vacuum wavelengths correspond very well to the energies at which the $n=0$ and the $n=1$ lower plasmon branches cross the glass (core) light line. Using a tip at the "wrong" wavelength results in measuring the intensity of the electric field just as using an uncoated tip. Our retarded calculations suggest a coupling of the cylindrical surface plasmon to propagating waves in the core material. The cylinder radius at the end of the tip is too small for waveguide modes in the core to be sustained, but further up the fiber is much thicker and the dispersion of propagating waves is just the glass light line. So choosing $\left(k_{z}, \omega\right)$ on this line seems to be the right injection condition, which was probably met in references 5 and 6 .

To estimate the influence of the conical shape of a real tip, in Fig.s 3 and 4 we study the effect of changing the core radius of the cylinder on the dispersion of the lowest order surface plasmon modes. The cladding thickness, that is the difference between the inner and the outer radius $d=a-b$, is kept constant in each plot. The intensity of the evanescent near field of a sample typically decreases within a few hundred nanometres in height. The tips used for the experiments in references 5 and 6 have a radius of $50 \mathrm{~nm}$ at the apex and an opening angle of $8^{\circ}$. So variing the core radius from $50 \mathrm{~nm}$ to $110 \mathrm{~nm}$ should approximately cover the part of the tip plunged into the near field. From Fig.3 we see that the lower $n=0$ branch changes very little with the core radius for $d=32 \mathrm{~nm}$ as well as for $d=24 \mathrm{~nm}$. Even if in Fig. $3 \mathrm{~b}$ the curve for the largest $b$ separates from the others for large $k_{z}$, the intersection with the glass light line is not affected by this shift. For $n=1$ (Fig.4) the upper branch is less dependent on the radius. Nevertheless, in Fig.4a $(d=32 \mathrm{~nm})$ the lower three curves cross the glass light line in the same energy range as all of them have already reached their final plateau. A broadening due to damping would very likely produce a good overlap for $\hbar c k_{z} \geq 3 \mathrm{eV}$. In Fig. $4 \mathrm{~b}(d=24 \mathrm{~nm})$ the intersection with the glass light line is on the first plateau to the left of the S-shape structure in the curves for $b=80 \mathrm{~nm}$ and $b=110 \mathrm{~nm}$, thus also at about the same energy. For the smallest radius $b=50 \mathrm{~nm}$, however, the dispersion curve gets considerably shifted to the left and therefore it crosses the glass light line at a higher energy. According to this estimate, if the $n=1$ surface plasmon mode plays a role in near-field detection as proposed in references 5 and 6 , the significant excitation takes place in a certain height range, i.e. at a certain radius, instead of being spread out all over the whole metallized tip. Of course, good contrast in near field images cannot be obtained without a somewhat limited range of the detection height. So the fact that the $n=1$ mode dispersion shifts more strongly with changing cylinder radius does not at all exclude this mode from having to be considered for understanding the properties of metal coated tips.

The crossing point between the $n=0$, the $n=1$ lower plasmon modes and the glass light line shifts with the cladding thickness. This is not only due to the S-shape structure in the curves, which stems from the dielectric function $\epsilon(\omega)$ for gold. Indeed, if we repeat the calculations from Fig.2 for silver in Fig.5, again, changing the metal thickness from $32 \mathrm{~nm}$ to $24 \mathrm{~nm}$ results in a perceptible shift of the energy at which the two lowest branches intersect the glass light line. 


\section{FIELD DISTRIBUTIONS}

\section{A. Radial Dependence}

As the angular and the $z$-dependence is the same and given by $S_{n}$ for all field components, in order to describe the field distributions of the eigenmodes, it suffices, in principle, to evaluate the radial parts from eqs.(1) which can be viewed as radius dependent amplitudes. In figures 6-9, we plot these amplitudes of the field components as a function of $r$ for the modes with $n=0,1$ and 2. As a normalization, we arbitrarily chose $\left|E_{z}\right|=1$ at the outside boundary of the metal. The numerical values for the field amplitudes are of the same order of magnitude for $\vec{E}$ and $\vec{H}$. Relatively to the electric field, the distribution of the magnetic field in any of the three regions (core, metal and outside) does not show any significantly higher intensity. (Equations are written in Gaussian units, so the numerical factor between $\vec{E}$ and $\vec{H}$ is $\epsilon^{1 / 2}$ for plane waves.) This presents an objection to the hypothesis, that gold coated fibre tips detect the magnetic instead of the electric field, can be explained in terms of overlap integrals. Approximating the tip by a cylinder, of course, is an oversimplification and more realistic tip shapes have to be studied. However, the resonance phenomenon associated with the extraordinary behavior of tips with certain cladding thicknesses detecting optical magnetic field intensities at specified wavelengths ${ }^{5,6}$ has to be in the metal ring region and not at the very end of the tip. Plasma resonances of full gold spheres or even metal spheres with dielectric core of the dimensions corresponding to the tip apex do not lie at the correct wavelengths.

Concerning the cylinder plasmon modes, we make the following observations: For the $n=0$ modes, of the six field components only $E_{z}, E_{r}$ and $H_{\theta}$ do not vanish (Fig.s 6and 9a). This mode resembles very much a surface plasmon on a planar metal film between two dielectrics, which only exists in p-polarization and has an electric field perpendicular to the metal surface (here $E_{r}$ ) and parallel to it in propagation direction (here $E_{z}$ ) as well as an inplane magnetic field perpendicular to the propagation direction (here $H_{\theta}$ ). Due to the negative dielectric function of the metal, the electric field perpendicular to the metal surfaces, that is $E_{r}$ in the case of the cylinder, has sign changes at the metal boundaries. All other field components of $\vec{E}$ and $\vec{H}$ are continuous. In Fig.6 the radius dependent amplitudes of the field components are plotted for three points on the lower $n=0$ dispersion branch from Fig.2a, in the region between the two dielectrics' light lines, on the glass light line and beyond it. Qualitatively the three distributions are alike. According to eqs.(5), in Fig.6b, $E_{z}$ is constant while $E_{r}$ and $H_{\theta}$ grow linearly with the radius inside the core. The field configuration for the point on the glass light line fits in between the other two and is equally obtained by approaching this point from either side. The same is true for the field distributions corresponding to the $n=1$ modes (Fig.7). There are only quantitative, but no qualitative differences whether $k_{c}$ is real, zero or imaginary. For completeness, we plot the radius dependent amplitudes of the field components for a point on the lower $n=2$ dispersion branch from Fig.2a in Fig.8 and examples for the high energy modes with $n=0,1$ and 2 in Fig.9. In contrast to the low energy modes, for the high energy modes, when going from the inside to the outside of the cylinder, the magnetic field components have no sign changes, whereas $E_{z}$ and $E_{\theta}$ have one more and $E_{r}$ has one less than for the low energy modes. As the frequencies of the upper modes lie mostly beyond the visible range, these plasmons are 
of little interest for applications in near-field optics.

\section{B. Angular Dependence}

To see the effect of the cylindrical geometry and of the phase factor $S_{n}$, the fields in a plane perpendicular to the cylinder axis, that is the fields resulting from $E_{r}$ and $E_{\theta}$ (resp. $H_{r}$ and $H_{\theta}$ ), are plotted as arrow diagrams in Fig.10 and the behavior of all three field components is explained schematically in Fig.11. With $\theta$ the fields vary as $e^{i n \theta}$ or, taking the real part, $\cos n \theta$. For the $n=0$ mode there is no angular dependence. On a circle of fixed radius, at a fixed height $(z=$ const.), at a given instant in time, each field component has a constant value. However, projecting the cylindrical field components in a Cartesian coordinate system for clearer illustration as done in Fig.11, we see that only the z-components are uniform in space. Due to the cylindrical geometry, for the radial and respectively the angular components, for every field vector there is one in opposite direction. The latter is also the case for the z-components of modes with $n>0$ as well as the radial and angular components of modes with $n \geq 2$ (Fig.11).

For $n=1$, the radial and angular fields on a circle of constant $r$ do not add up to zero and this has an important consequence: Because of the angular modulation following $e^{i \theta}$ for $n=1$ the radial and the angular field components can well have finite amplitudes at $r=0$ (Fig.s 7 and 9b) where the directions of the unit vectors $\vec{e}_{r}$ and $\vec{e}_{\theta}$ in cylindrical coordinates get undefined. Integrating the radial or the angular component vector over a circle as shown in Fig.11b, we see that, even in the limit where the radius of the circle approaches zero $(r \rightarrow 0)$, a field vector in the $\mathrm{x}-\mathrm{y}$-plane rests in each case. A possible phase shift between the components has been omitted in Fig.11b; all components are drawn as having their maximum positive value at $\theta=0^{\circ}$. At this stage we have to add that all the coefficients $a_{n}^{j}$, $b_{n}^{j}, \alpha_{n}^{c}$ and $\beta_{n}^{c}$ for all $n$ come out either real or purely imaginary. (We only implied Bessel, no Hankel functions, and $\epsilon(\omega)$ for the metal was also taken as real.) The radial parts of the field components, i.e. the expressions from eqs.(1) resp. eqs.(4) without the factor $S_{n}$ also turn out to be either real or purely imaginary, but not complex. That is, phase shifts between the field components are multiples of $\pi / 2$. A consistent scheme is given by:

$$
\begin{array}{lll}
E_{z} \text { imaginary, } & E_{\theta} \text { imaginary, } & E_{r} \text { real } \\
H_{z} \text { real, } & H_{\theta} \text { real, } & H_{r} \text { imaginary }
\end{array}
$$

$E_{z}, E_{\theta}$ and $H_{r}$ in figures 6-9 have to be multiplied by the imaginary unit $i$, but otherwise the relative signs of the components are given consistently. This information about the relative phase is necessary for the vector addition of $E_{r}$ and $E_{\theta}$ (resp. $H_{r}$ and $H_{\theta}$ ) as done in Fig.10. $n=1$ is the only $n$ value for which the radial and angular field components do not vanish at $r=0$. Note also from Fig. 7 that for both the electric and the magnetic field the amplitudes of the radial and the angular component are of equal absolute value towards the center of the cylinder. Combining the radial and the angular component of the electric (or the magnetic) field with the correct phase between them, we find that they reinforce each other as depicted in Fig.11d. Furthermore, in the middle of the core we find a phase difference of $\pi / 2$ between the electric and the magnetic field in the plane perpendicular to the $z$-axis (see Fig.s 10c and d). Therefore, in the center of the core, where the $z$-components 
of the fields vanish, the $n=1$ plasmon mode is nearly a circularly polarized wave travelling in the $z$-direction. For the special case that there is no radial wave vector this is easily seen from eqs.(4) as for $n=1$ the $\beta$-terms describe a plane wave in the core material. So exciting the $n=1$ plasmon mode at an energy corresponding to the glass light line should provide good signal injection from the tip into the fibre. The $z$-component of the electric field of the $n=0$ plasmon mode has no overlap with a transverse wave propagating in $z$-direction in the core material.

Because of the phase factor $S_{n}$ the field patterns like the ones drawn in Fig.10a-f turn around the $z$-axis with time. However, this is merely due to the choice of basis of angular eigenfunctions. By building linear combinations out of terms with both signs of $\theta$ in eq.(2), $e^{i n \theta}$ and $e^{-i n \theta}$, we cuold change from right and left turning circularly polarized (surface plasmon) waves to "linearly" polarized ones, depending on $\theta$ as $\cos n \theta$ or $\sin n \theta$. In this basis, the field patterns in Fig.10 would be viewed as oscillating in time, but with their directions and characteristic axis resting fixed in space.

Figure 10 illustrates that, at least concerning the fields in a plane perpendicular to the cylinder axis, the $n=0$ plasmon mode has monopole character, the $n=1$ mode dipole character, the $n=2$ mode quadrupole character, etc. For $n=1$ the dipole is probably more easily recognized in the magnetic field than in the electric field. The magnetic field in Fig.10d points upwards inside the cylinder and field lines are closed via the outside with the strongest downward pointing fields on the $\mathrm{x}$-axis. This field distribution resembles more the pattern of a dipole with its loops of field lines than the electric field distribution of the plasmon. Here the fields are opposed inside and outside the cylinder on the dipole axis (x-axis in Fig.10c) and also, outside the cylinder the fields nearly vanish on the perpendicular axis (y-axis in Fig.10c). This observation should, however, only be taken as a qualitative remark.

\section{Dipole Moments}

As our cylinders are of subwavelength diameter, coupling of their eigenmodes to an external field will in a first approximation be descibed by the dipole terms ${ }^{10}$

$$
\vec{p}_{\text {eff }} \vec{E}_{\text {ext }}+\vec{m}_{\text {eff }} \vec{B}_{\text {ext }}
$$

where $\vec{p}_{e f f}$ and $\vec{m}_{e f f}$ are the effective electric and magnetic dipole moments of the cylinder

mode and $\vec{E}_{\text {ext }}$ and $\vec{B}_{\text {ext }}$ are the external electric and magnetic field. As seen from the field distributions in the last section, the $n=1$ cylinder plasmon mode contains an electric as well as a magnetic dipole. In the following we calculate the electric and the magnetic dipole moment ${ }^{10}$

$$
\begin{gathered}
\vec{p}=-\frac{1}{i \omega} \int \vec{J}(\vec{x}) d^{3} x \\
\vec{m}=\frac{1}{2 c} \int(\vec{x} \times \vec{J}(\vec{x})) d^{3} x
\end{gathered}
$$

(where $\vec{x}$ is the position vector) for a plasmon mode for a slice of unit length of the cylinder. Thus we suppress the integration over $z$ in (6) and (7) and take $\vec{p}$ and $\vec{m}$ per unit length of the cylinder. As we have put all information about the materials into $\epsilon$, there are no free 
charges or currents. For harmonic time dependent fields the current is thus the polarization current given in the Gaussian system by

$$
\vec{J}=\frac{1}{4 \pi}(\epsilon-1) \vec{E}
$$

The position vector $\vec{x}$ is $r \vec{e}_{r}$ in cylindrical coordinates. Inversely, the unit vectors $\vec{e}_{r}$ and $\vec{e}_{\theta}$ projected into Cartesian coordinates are

$$
\begin{aligned}
& \vec{e}_{r}=\vec{e}_{x} \cos \theta+\vec{e}_{y} \sin \theta \\
& \vec{e}_{\theta}=-\vec{e}_{x} \sin \theta+\vec{e}_{y} \cos \theta
\end{aligned}
$$

Furthermore, we write

$$
E_{r}(r, \theta, z)=E_{r}(r) \exp \left(i n \theta+i k_{z} z-i \omega t\right)=E_{r}(r)(\cos n \theta+i \sin n \theta) \exp \left(i k_{z} z-i \omega t\right)
$$

where $E_{r}$ with argument $r$ instead of $r, \theta$ and $z$ is the radial part from eqs.(1) resp. eqs.(4), that is $E_{r}(r, \theta, z)$ without the factor $S_{n}$. The electric dipole moment of a plasmon mode per unit length of the cylinder is then

$$
\begin{aligned}
\vec{p} & =-\frac{1}{i \omega} \int_{0}^{2 \pi} d \theta \int_{0}^{a} r d r \vec{J} \\
& =-\frac{1}{i \omega} \int_{0}^{2 \pi} d \theta \int_{0}^{a} r d r \frac{\epsilon(r)-1}{4 \pi} \exp \left(i n \theta+i k_{z} z-i \omega t\right)\left(E_{r}(r) \vec{e}_{r}+E_{\theta}(r) \vec{e}_{\theta}+E_{z}(r) \vec{e}_{z}\right) \\
& =-\frac{\exp \left(i k_{z} z-i \omega t\right)}{4 \pi i \omega} \int_{0}^{2 \pi} d \theta \int_{0}^{a} r d r(\epsilon(r)-1)(\cos n \theta+i \sin n \theta) \\
& \left(E_{r}(r)\left(\cos \theta \vec{e}_{x}+\sin \theta \vec{e}_{y}\right)+E_{\theta}(r)\left(-\sin \theta \vec{e}_{x}+\cos \theta \vec{e}_{y}\right)+E_{z}(r) \vec{e}_{z}\right) \\
& =-\frac{\exp \left(i k_{z} z-i \omega t\right)}{4 i \omega} \delta_{n, 1}\left(\vec{e}_{x}+i \vec{e}_{y}\right) \int_{0}^{a} d r r(\epsilon(r)-1)\left(E_{r}(r)-i E_{\theta}(r)\right)
\end{aligned}
$$

$\delta_{n, 1}$ in eq.(12) indicates that $\vec{p}$ only exists for $n=1$. (The kind of "dipole" presented by $E_{z}$ for $n=0$ is not described by regarding only a thin slice of the cylinder at constant $z$, because here the charge separation is in $z$-direction. Anyway, as there is no $H_{z}$-component, the $n=0$-mode has no magnetic dipole moment the electric one could be compared to.) As $E_{r}(r)$ is real and $E_{\theta}(r)$ is imaginary, $E_{r}(r)-i E_{\theta}(r)$ is real and corresponds to adding the curves for these two amplitudes in Fig.7.

The magnetic dipole moment $\vec{m}$ is calculated in the same manner as the electric dipole moment $\vec{p}$. We assume here, that the outside medium is air with $\epsilon_{a}=1$, so that $\vec{J}$ vanishes for $r>a$. An outside medium with a dielectric constant different from 1 and thus the integration over $r$ up to infinity would, however, cause no problem, because the exponential decay of the fields outside the cylinder would assure that the values of the integrals rest finite.

$$
\vec{m}=\frac{1}{2 c} \int_{0}^{2 \pi} d \theta \int_{0}^{a} r d r \vec{x} \times \vec{J}
$$




$$
\begin{aligned}
& =\frac{1}{2 c} \int_{0}^{2 \pi} d \theta \int_{0}^{a} r d r \frac{\epsilon(r)-1}{4 \pi} \exp \left(i n \theta+i k_{z} z-i \omega t\right) \\
& r \vec{e}_{r} \times\left(E_{r}(r) \vec{e}_{r}+E_{\theta}(r) \vec{e}_{\theta}+E_{z}(r) \vec{e}_{z}\right) \\
& =\frac{\exp \left(i k_{z} z-i \omega t\right)}{8 c} \delta_{n, 1}\left(i \vec{e}_{x}-\vec{e}_{y}\right) \int_{0}^{a}(\epsilon(r)-1) r^{2} E_{z}(r) d r
\end{aligned}
$$

$\epsilon(r)$ is understood to be $\epsilon_{c}$ for $r<b, \epsilon_{m}$ for $b<r<a$ and $\epsilon_{a}$ for $r>a$. As before, $E_{r}(r), E_{\theta}(r)$ and $E_{z}(r)$ are the radius dependent amplitudes of the field components, that is the expressions from eqs.(1) resp. eqs.(4) without $S_{n} . \vec{m}$ also only exists if $n=1$. Like $\vec{E}$ and $\vec{H}$, in the Gaussian system $\vec{p}$ and $\vec{m}$ are in the same units and directly comparable. $|\vec{p}|$ and $|\vec{m}|$ following the lower $n=1$ dispersion branch of Fig.2a are plotted in Fig.12 and some values are listed some in table 1 . Other than for atoms, where the magnetic dipole moment usually is several orders of magnitude less than the electric dipole moment in the optical range of frequencies ${ }^{11}$, for surface plasmons on a cylinder, whose circumference is about a wavelength, the electric and the magnetic dipole moment become comparable. $|\vec{m}|$ is not always bigger than $|\vec{p}|$, though. For the parameters chosen in Fig.12 $(a=112 \mathrm{~nm}$ and $b=80 \mathrm{~nm})|\vec{m}|$ exceeds $|\vec{p}|$ for $\hbar c k_{z} \geq 2.7 \mathrm{eV}$. So at the intersection of the plasmon dispersion with the core light line at $\hbar c k_{z}=3.2 \mathrm{eV}$ the plasmon's magnetic dipole moment is greater than its electric one. For the parameters from Fig.2b $(a=104 \mathrm{~nm}, b=80 \mathrm{~nm})|\vec{m}|$ and $|\vec{p}|$ are just found to be equal where the low energy $n=1$ dispersion branch crosses the glass light line at $\hbar c k_{z}=2.7 \mathrm{eV} ;|\vec{m}|>|\vec{p}|$ for greater $k_{z}$ and $|\vec{m}|<|\vec{p}|$ to the left of the glass light line. The curves in Fig.12 do not change much, if just $a$ is varied. However, changing $b$ from $80 \mathrm{~nm}$ to $110 \mathrm{~nm}$ while keeping $a-b$ constant at $24 \mathrm{~nm}$ (see Fig.4b) shifts the intersection between $|\vec{m}|$ and $|\vec{p}|$ to $\hbar c k_{z}=1.8 \mathrm{eV}$, so that at $\hbar c k_{z}=2.7 \mathrm{eV}$ where the plasmon dispersion meets the glass light line, $|\vec{m}|$ is considerably larger than $|\vec{p}|$. Keeping in mind that even the metal ring region of the tip with about the correct radius for surface plasmon excitation at a given frequency has a certain opening angle, our calculations are consistent with the fact that, as for tips with $32 \mathrm{~nm}$ gold cladding resonant a $543 \mathrm{~nm}$, also for tips with $24 \mathrm{~nm}$ cladding resonant at $633 \mathrm{~nm}^{5,6}$, the cylindrical surface plasmon's magnetic dipole moment can well exceed its electric one. Of the height range sustaining the surface plasmon, the part with a little greater radius a little further up the tip may even be more important than the lower end as the farther is nearer to the signal injection into the actual fibre. For the cylinder, as a thumb rule, we may deduce, that the plasmon dipole is more of magnetic than of electric type as long as the inner metal circumference is larger than the wavelength.

\section{CONCLUSIONS}

We have presented fully retarded calculations for the non-radiative surface plasmon modes on metal cylinders with dielectric core. The dispersion as well as the distributions of the electric and the magnetic field of these eigenmodes have been detailed. We associate the resonance phenomenon leading to the measurement of the magnetic field by metal cladded near-field tips reported in references 5 and 6 to the excitation of the low energy $n=1$ cylindrical plasmon mode and the special condition that the plasmon frequency and the wave 
vector along the cylinder axis coincide with the light line of the core material of the tip. The plasmon excited at the cladded end of an optical fibre can only lead to a signal transmitted through the fibre if the frequency and the wave vector coincide with those of propagating waves in the fibre core material. The low energy $n=0$ surface plasmon mode nearly coincides with the $n=1$ mode for metal cylinders with dielelectric core for wave vectors near and beyond the core light line. But as the $n=0$ mode has no overlap with propagating waves in the core material, it is unlikely to be of importance for the detection in PSTM. For the $n=1$-mode we find that effective electric and magnetic dipole moments per unit length of the cylinder are of the same order of magnitude. For parameters corresponding to the tip dimensions from references 5 and 6, the magnetic dipole moment is found to exceed the electric one, which can be related to the fact that cladded tips at certain frequencies image the optical magnetic near-field. Of course, the infinitely long cylinder is a very crude model for near-field tips, and in a PSTM setup, the distance between the sample and the ring region of the tip is not really large compared to the tip diameter. So firstly, more realistic model geometries have to be studied, and secondly, the surface plasmon mode excitation should be decribed more exactly than in the dipole approximation.

\section{ACKNOWLEDGMENTS}

The Regional Council of Burgundy provided financial support to this work. 


\section{REFERENCES}

${ }^{1}$ H. Raether, Surface Plasmons, Springer Tracts in Modern Physics 111, Springer 1988.

2 E.N.Economou, Phys. Rev. 182, 439 (1969).

${ }^{3}$ J. Koglin, U. C. Fischer and H. Fuchs, Phys. Rev. B 55, 7977 (1997).

${ }^{4}$ L. Novotny, D. W. Pohl and B. Hecht, Ultramicroscopy 61, 1 (1995).

${ }^{5}$ E. Devaux, A. Dereux, E. Bourillot, J.-C. Weeber, Y. Lacroute, J.-P. Goudonnet and C. Girard, Appl. Surf. Sci. 164, 124 (2000).

${ }^{6}$ E. Devaux, A. Dereux, E. Bourillot, J.-C. Weeber, Y. Lacroute, J.-P. Goudonnet and C. Girard, Phys. Rev. B 62, 10504 (2000).

${ }^{7}$ E. D. Palik (ed), Handbook of Optical Constants of Solids, Academic Press 1998.

${ }^{8}$ C. A. Pfeiffer, E. N. Economou and K. L. Ngai, Phys. Rev. B 10, 3038 (1974).

${ }^{9}$ L. Novotny and C. Hafner, Phys. Rev. E 50, 4094 (1994).

${ }^{10}$ J. D. Jackson, Classical Electrodynamics, Wiley 1975, chapter 9.

${ }^{11}$ L.Landau et E.Lifchitz, Physique Théorique, Vol.VIII, Moscow 1969. 


\section{TABLES}

\begin{tabular}{|c|c|c|c|c|c|}
\hline \hline $\mathrm{a}(\mathrm{nm})$ & $\mathrm{b}(\mathrm{nm})$ & $\hbar c k_{z}(\mathrm{eV})$ & $\hbar \omega(\mathrm{eV})$ & $|\vec{p}|$ (a.u.) & $|\vec{m}|$ (a.u.) \\
\hline 112 & 80 & 2.0 & 1.85 & $1.84 * 10^{-30}$ & $1.27 * 10^{-30}$ \\
112 & 80 & 3.2 & 2.27 & $0.50 * 10^{-30}$ & $0.85 * 10^{-30}$ \\
112 & 80 & 4.5 & 2.37 & $0.12 * 10^{-30}$ & $0.60 * 10^{-30}$ \\
\hline 104 & 80 & 2.0 & 1.82 & $1.71 * 10^{-30}$ & $1.08 * 10^{-30}$ \\
104 & 80 & 2.7 & 1.92 & $0.82 * 10^{-30}$ & $0.79 * 10^{-30}$ \\
104 & 80 & 4.5 & 2.32 & $0.13 * 10^{-30}$ & $0.51 * 10^{-30}$ \\
\hline 134 & 110 & 2.0 & 1.76 & $1.94 * 10^{-30}$ & $2.34 * 10^{-30}$ \\
134 & 110 & 2.7 & 1.87 & $0.70 * 10^{-30}$ & $1.58 * 10^{-30}$ \\
134 & 110 & 4.5 & 2.30 & $0.06 * 10^{-30}$ & $0.91 * 10^{-30}$ \\
\hline \hline
\end{tabular}

TABLE I. Table 1. Electric and magnetic dipole moments for some points on the low energy $n=1$ dispersion branches from Fig.2. 


\section{FIGURES}

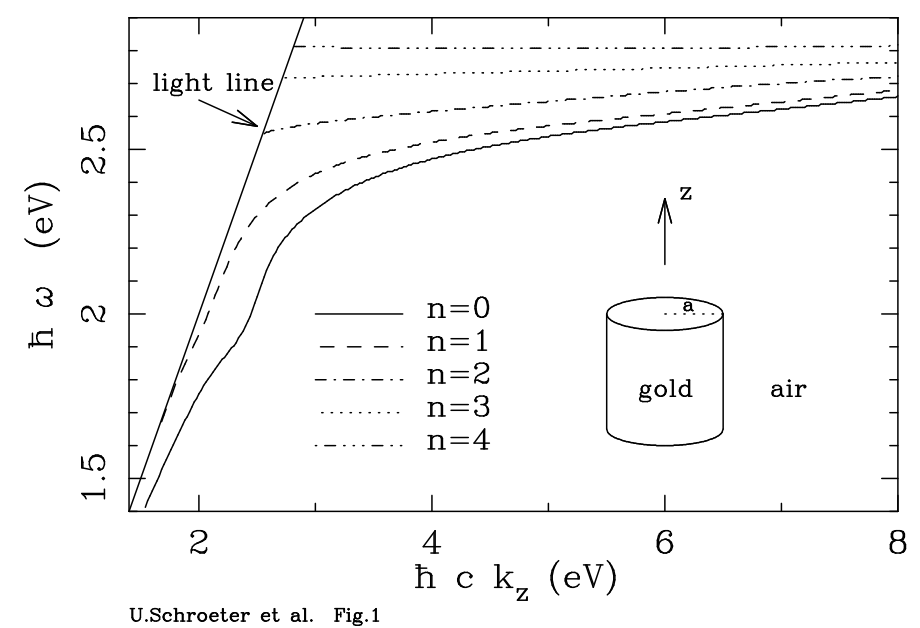

FIG. 1. Dispersion of the first five non-radiative surface plasmon modes of a gold cylinder of radius $a=100 \mathrm{~nm}$ surrounded by air. 

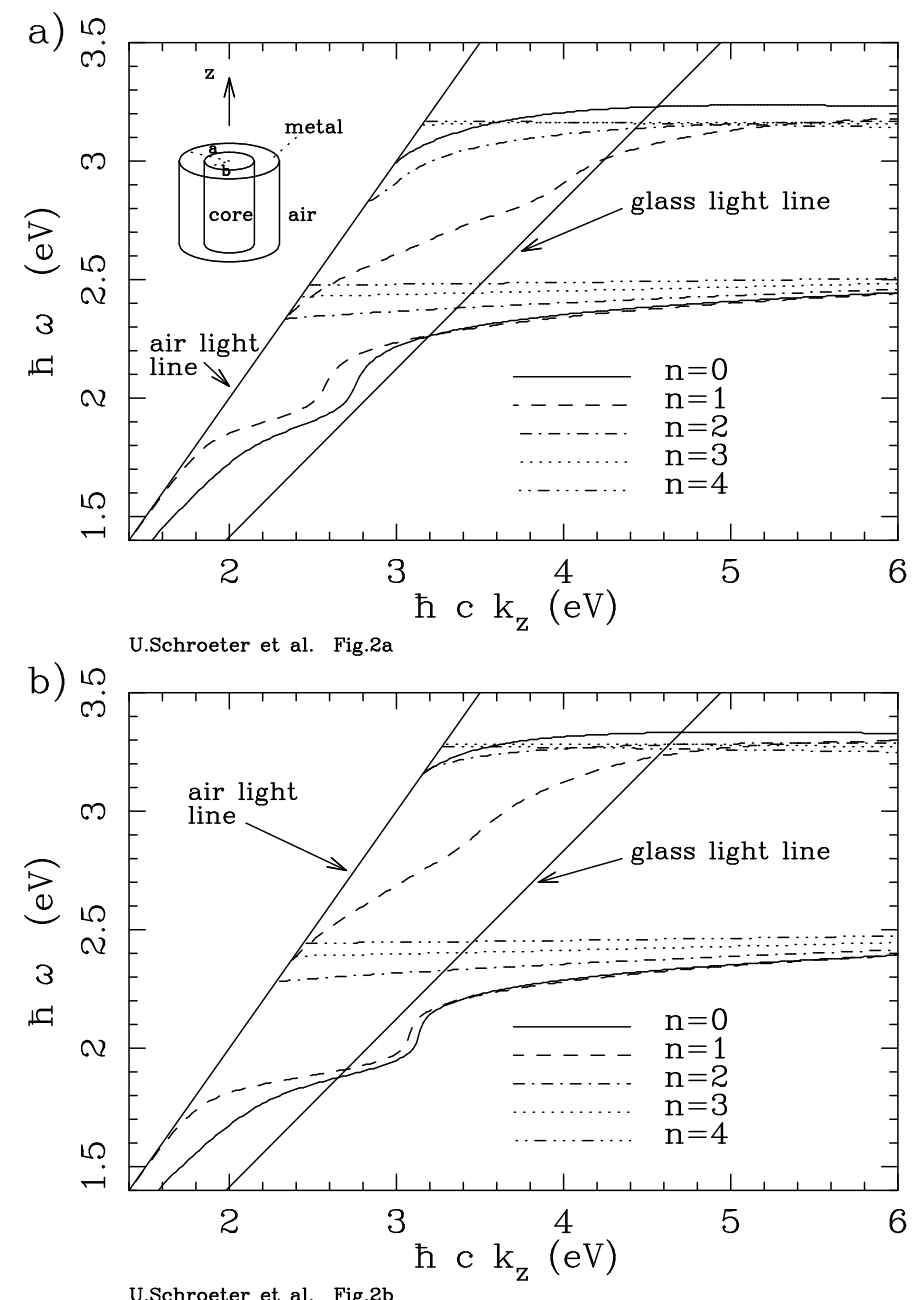

FIG. 2. Surface plasmon dispersion up to 4 th order branches for gold cylinders with dielectric core $\left(\epsilon_{c}=2\right)$ embedded in air $\left(\epsilon_{a}=1\right)$. (a) inner radius $b=80 \mathrm{~nm}$, outer radius $a=112 \mathrm{~nm}$. (b) inner radius $b=80 \mathrm{~nm}$, outer radius $a=104 \mathrm{~nm}$. 

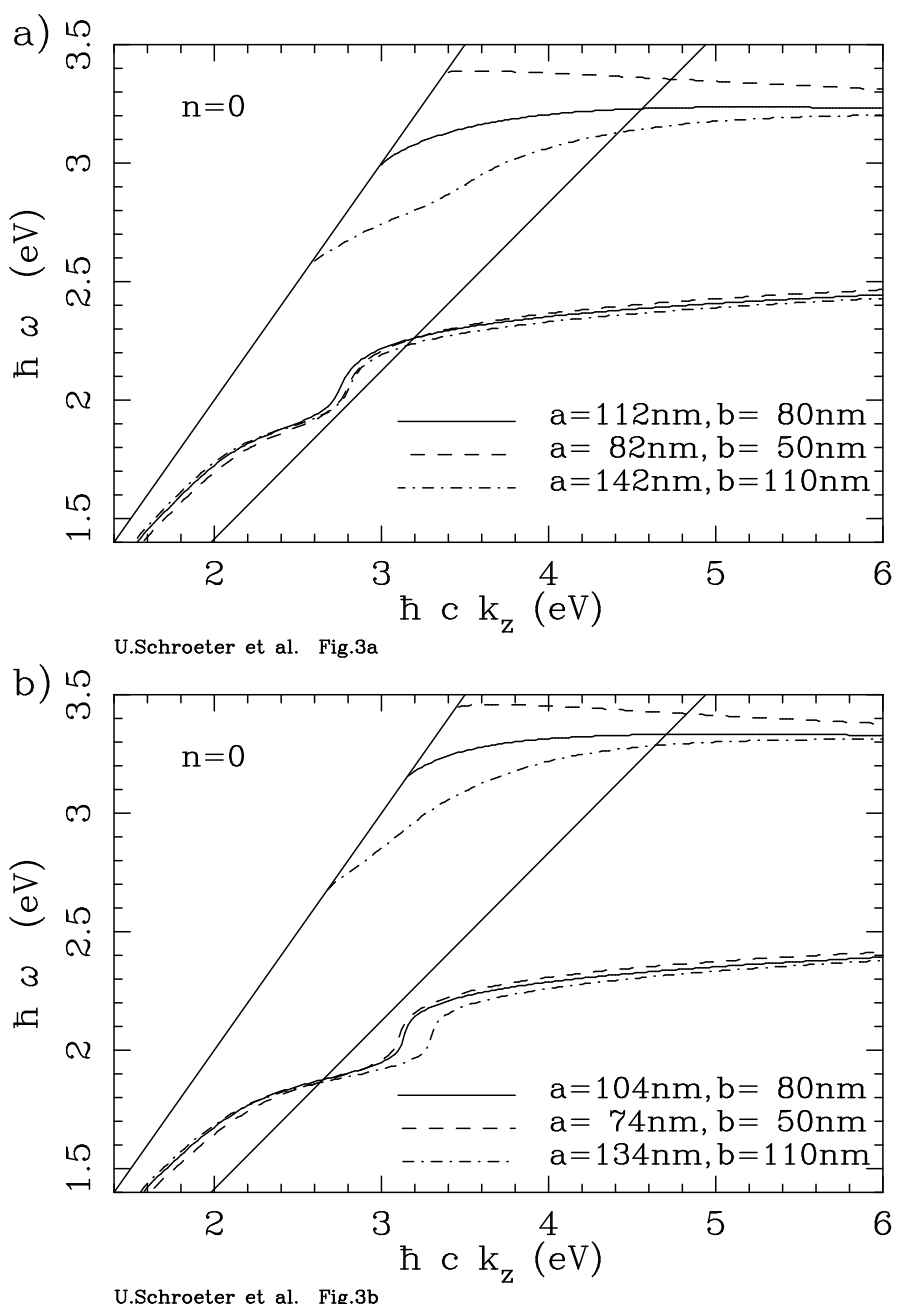

FIG. 3. Dispersion of the $n=0$ modes as a function of the cylinder radius keeping the metal cladding thickness constant. (a) $a-b=32 \mathrm{~nm}$, (b) $a-b=24 \mathrm{~nm}$. 

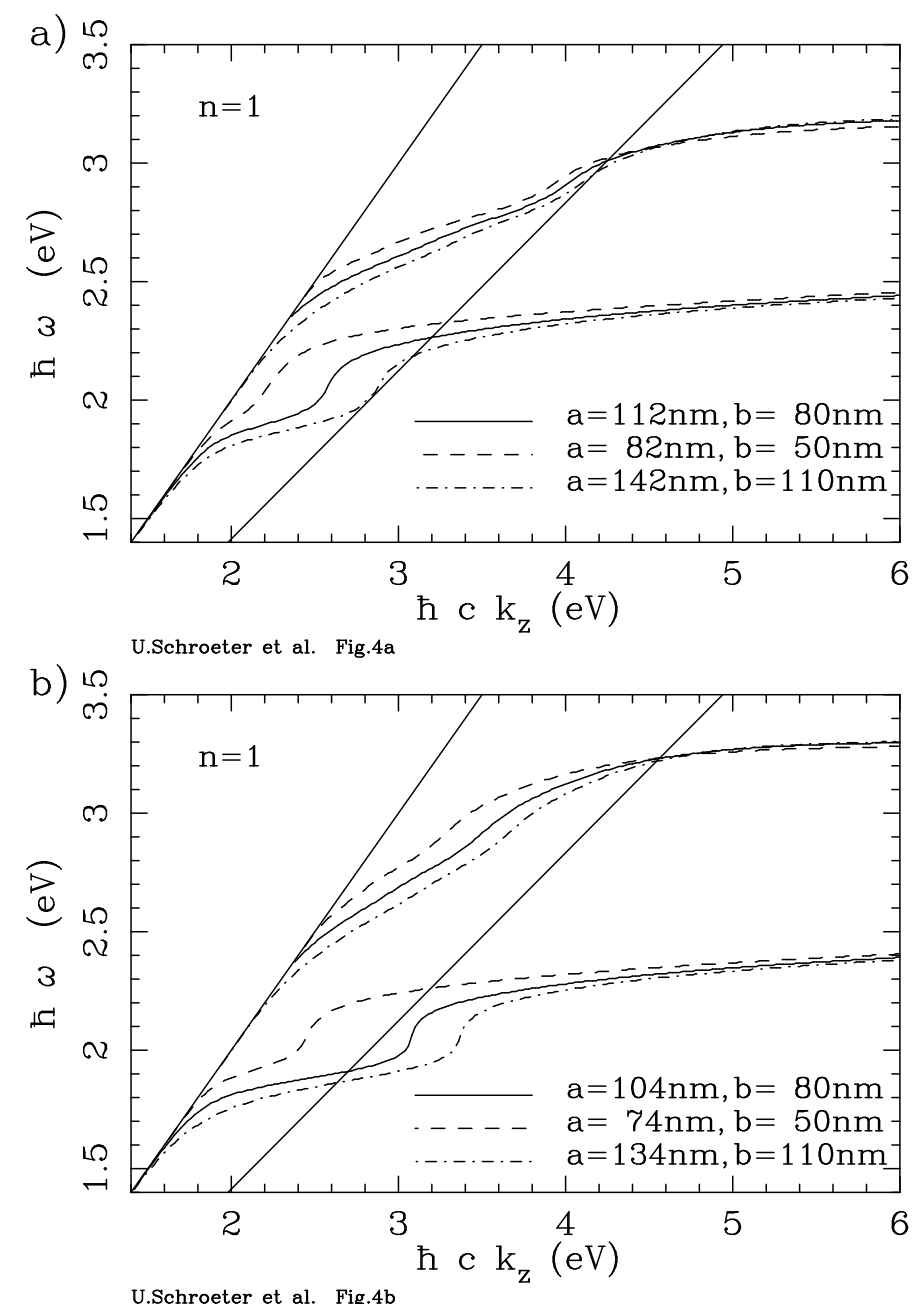

FIG. 4. Dispersion of the $n=1$ modes as a function of the cylinder radius keeping the metal cladding thickness constant. (a) $a-b=32 \mathrm{~nm}$, (b) $a-b=24 \mathrm{~nm}$. 

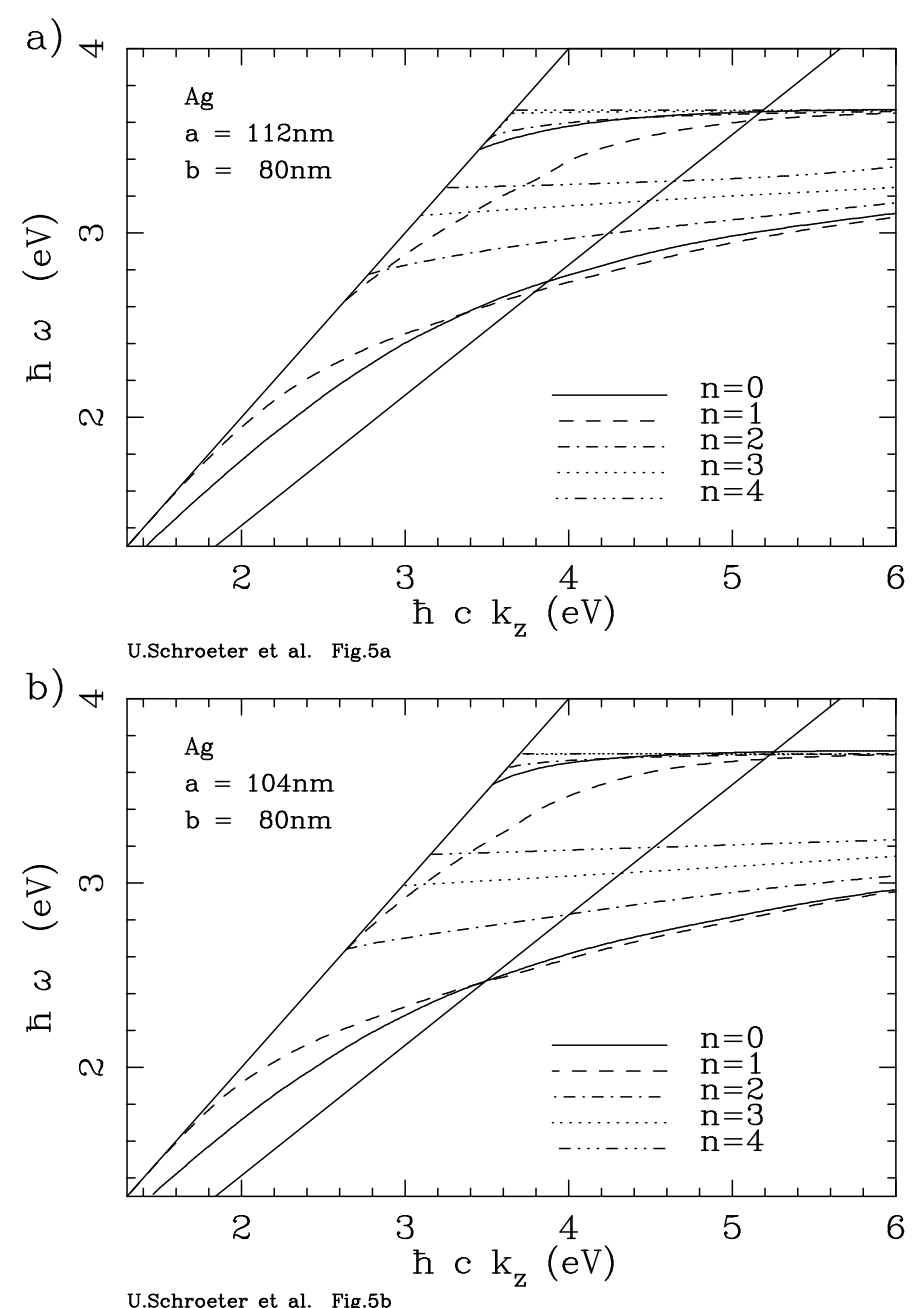

FIG. 5. Surface plasmon dispersion up to 4 th order branches for silver cylinders with dielectric core $\left(\epsilon_{c}=2\right)$ embedded in air $\left(\epsilon_{a}=1\right)$. (a) inner radius $b=80 \mathrm{~nm}$, outer radius $a=112 \mathrm{~nm}$. (b) inner radius $b=80 \mathrm{~nm}$, outer radius $a=104 \mathrm{~nm}$. 

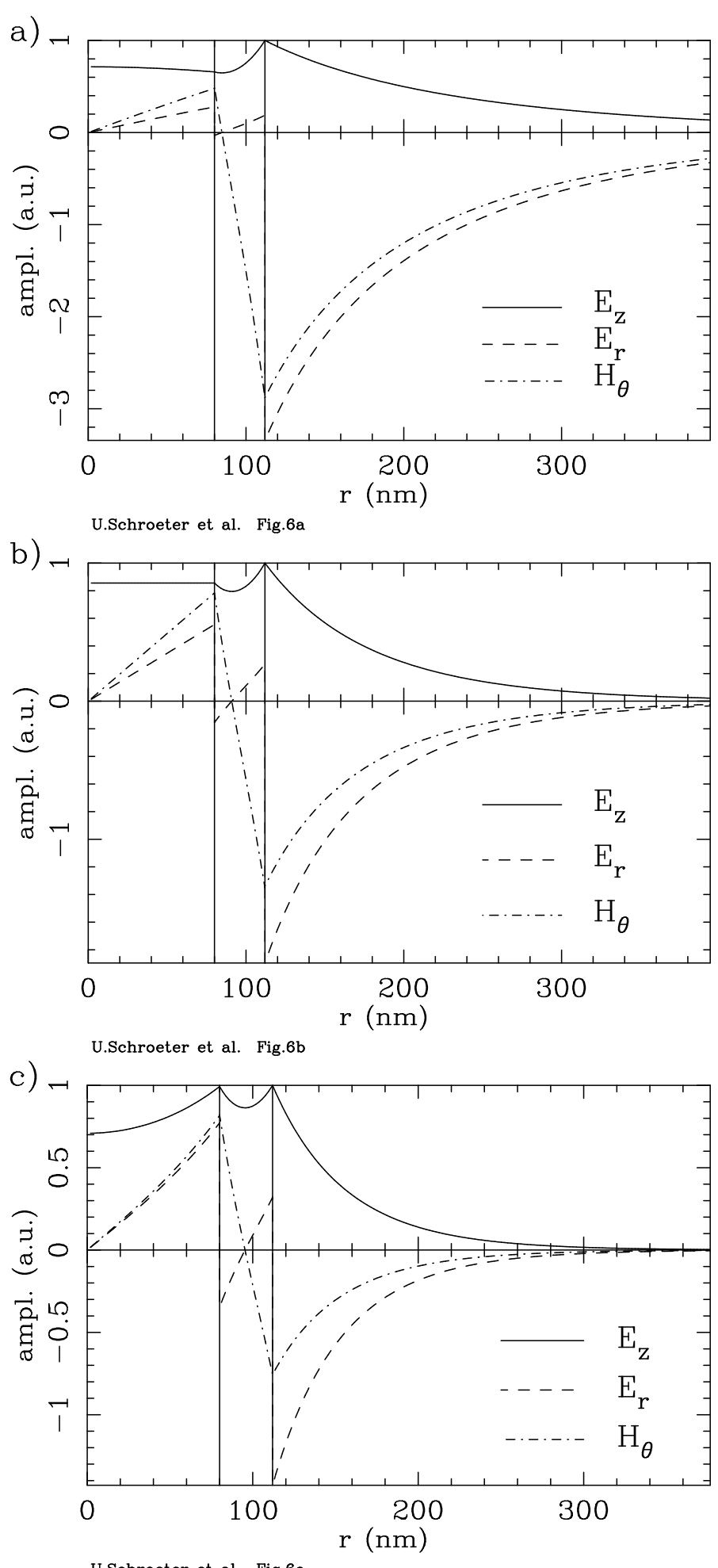

U.S. 6. Radial dependence of the field components for the lower $n=0$ mode from Fig. $2 \mathrm{a}$, that is cylinder radii $a=112 \mathrm{~nm}$ and $b=80 \mathrm{~nm}$. (a) $\hbar c k_{z}=2.0 \mathrm{eV}, \hbar \omega=1.72 \mathrm{eV}$. (b) $\hbar c k_{z}=3.2 \mathrm{eV}, \hbar \omega=2.26 \mathrm{eV}$. (c) $\hbar c k_{z}=4.5 \mathrm{eV}, \hbar \omega=2.38 \mathrm{eV}$. The vertical lines mark the core radius $b$ and the outside cylinder radius $a$. 

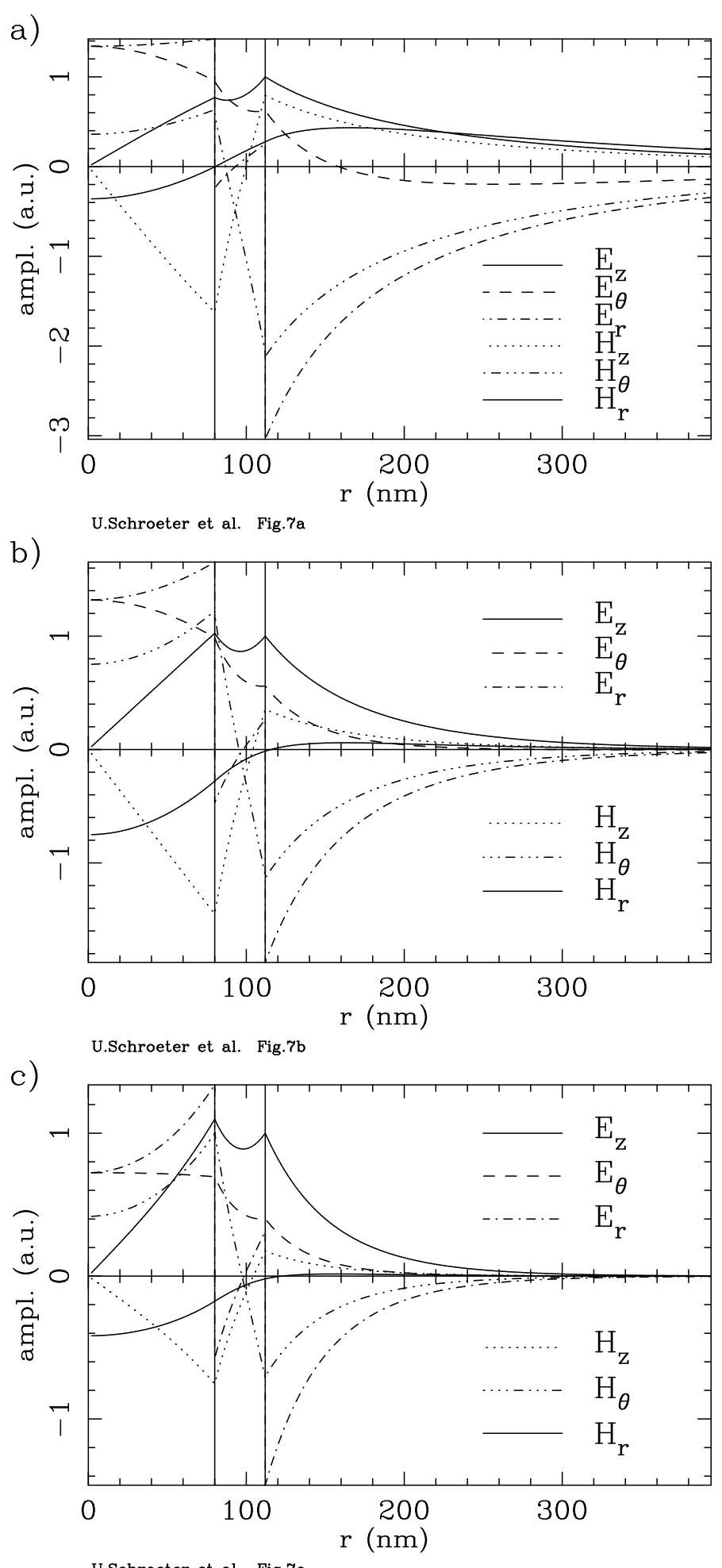

FIG. 7. Radial dependence of the field components for the lower $n=1$ mode from Fig.2a, that is cylinder radii $a=112 \mathrm{~nm}$ and $b=80 \mathrm{~nm}$. (a) $\hbar c k_{z}=2.0 \mathrm{eV}, \hbar \omega=1.85 \mathrm{eV}$. (b) $\hbar c k_{z}=3.2 \mathrm{eV}, \hbar \omega=2.27 \mathrm{eV}$. (c) $\hbar c k_{z}=4.5 \mathrm{eV}, \hbar \omega=2.37 \mathrm{eV}$. Of the two solid curves in each plot $H_{r}$ is the one whose slope doesn't change at $r=b$ and $r=a$. 


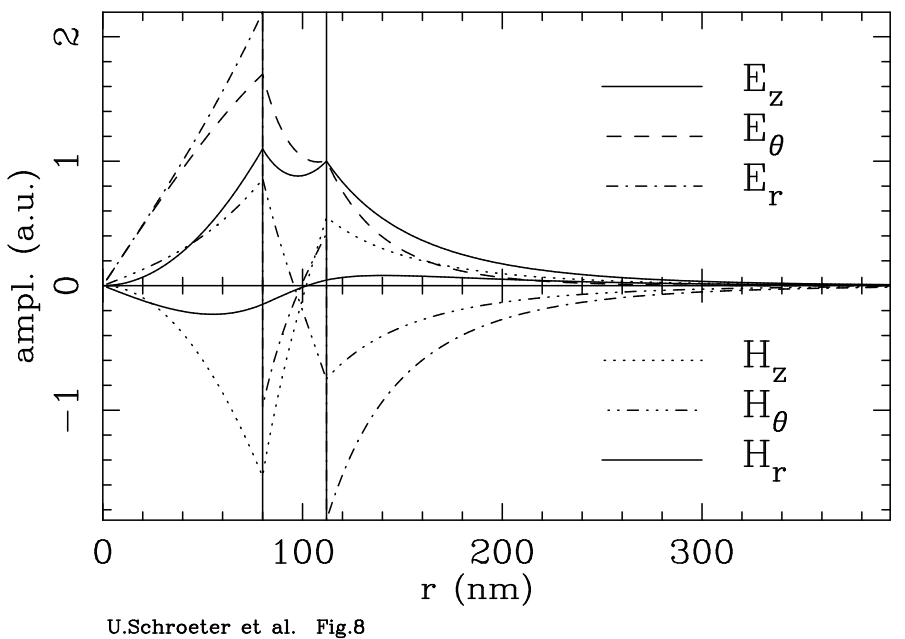

FIG. 8. Radial dependence of the field components for the lower $n=2$ mode from Fig.2a, that is cylinder radii $a=112 \mathrm{~nm}$ and $b=80 \mathrm{~nm}$. $\hbar c k_{z}=3.4 \mathrm{eV}, \hbar \omega=2.38 \mathrm{eV}$. 

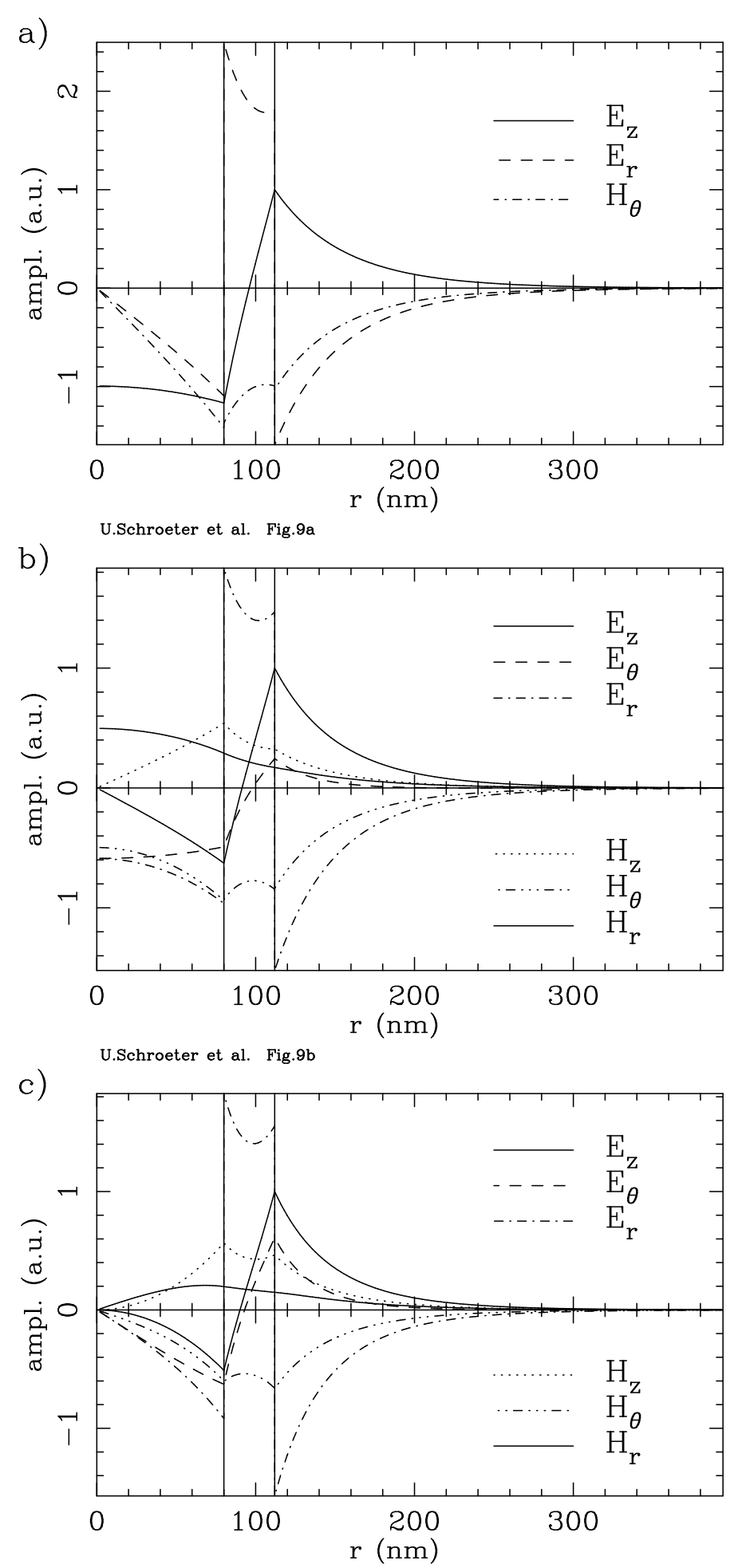

U.Schroeter et al. Fig.9c $a=112 \mathrm{~nm}$ and $b=80 \mathrm{~nm}$. (a) $n=0, \hbar c k_{z}=5.0 \mathrm{eV}, \hbar \omega=3.24 \mathrm{eV}$. (b) $n=1, \hbar c k_{z}=5.0 \mathrm{eV}, \hbar \omega=3.12 \mathrm{eV}$. (c) $n=2, \hbar c k_{z}=5.0 \mathrm{eV}, \hbar \omega=3.16 \mathrm{eV}$. 
a)

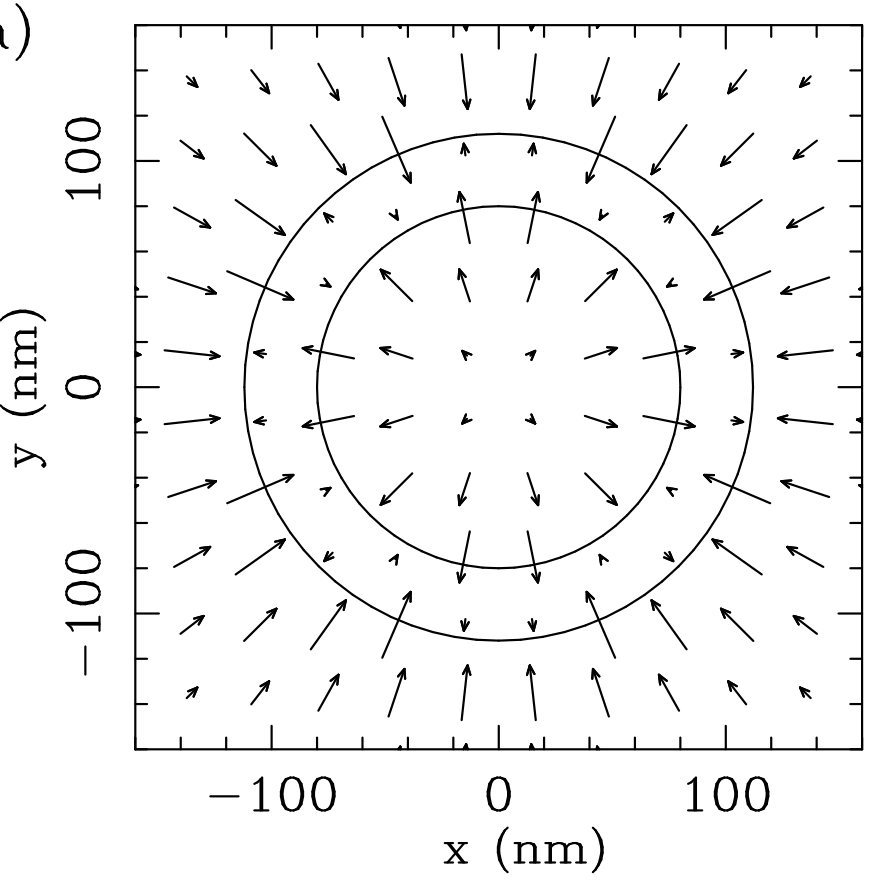

b)

U.Schroeter et al. Fig.10a

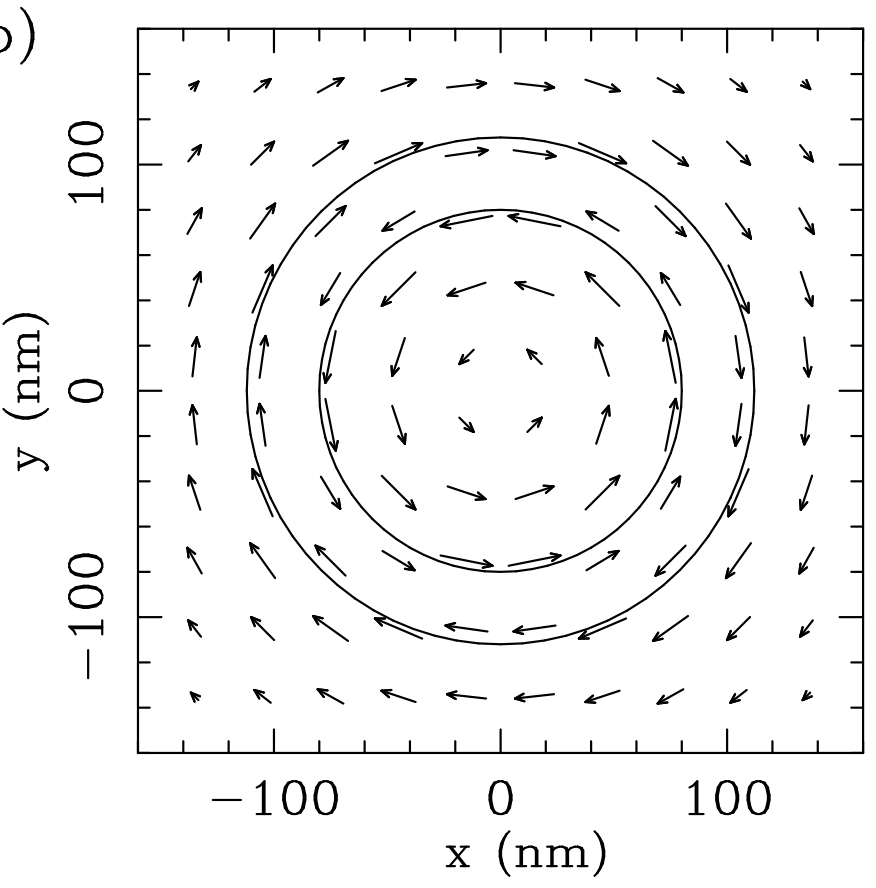

U.Schroeter et al. Fig.10b 

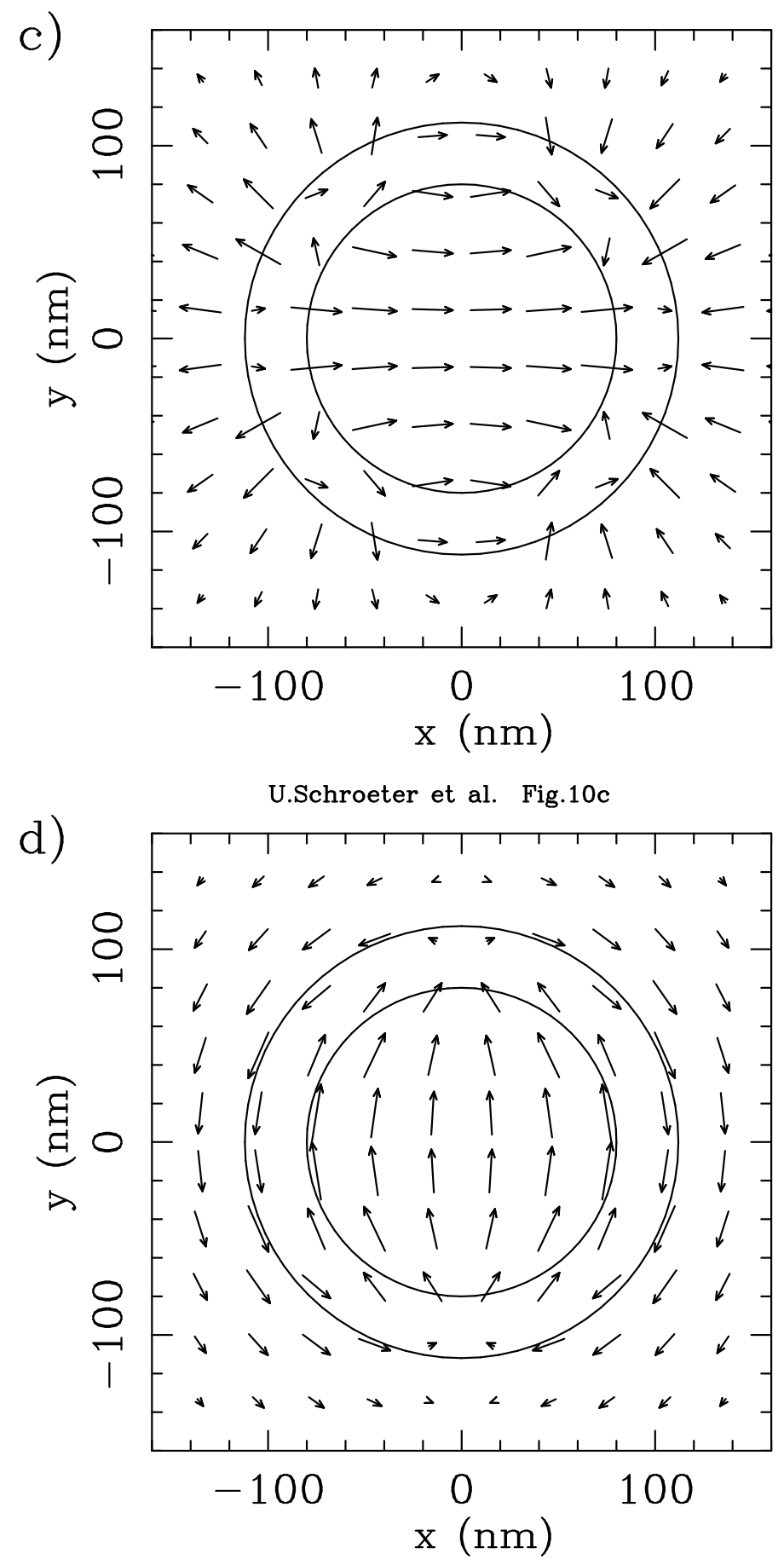

U.Schroeter et al. Fig.10d 

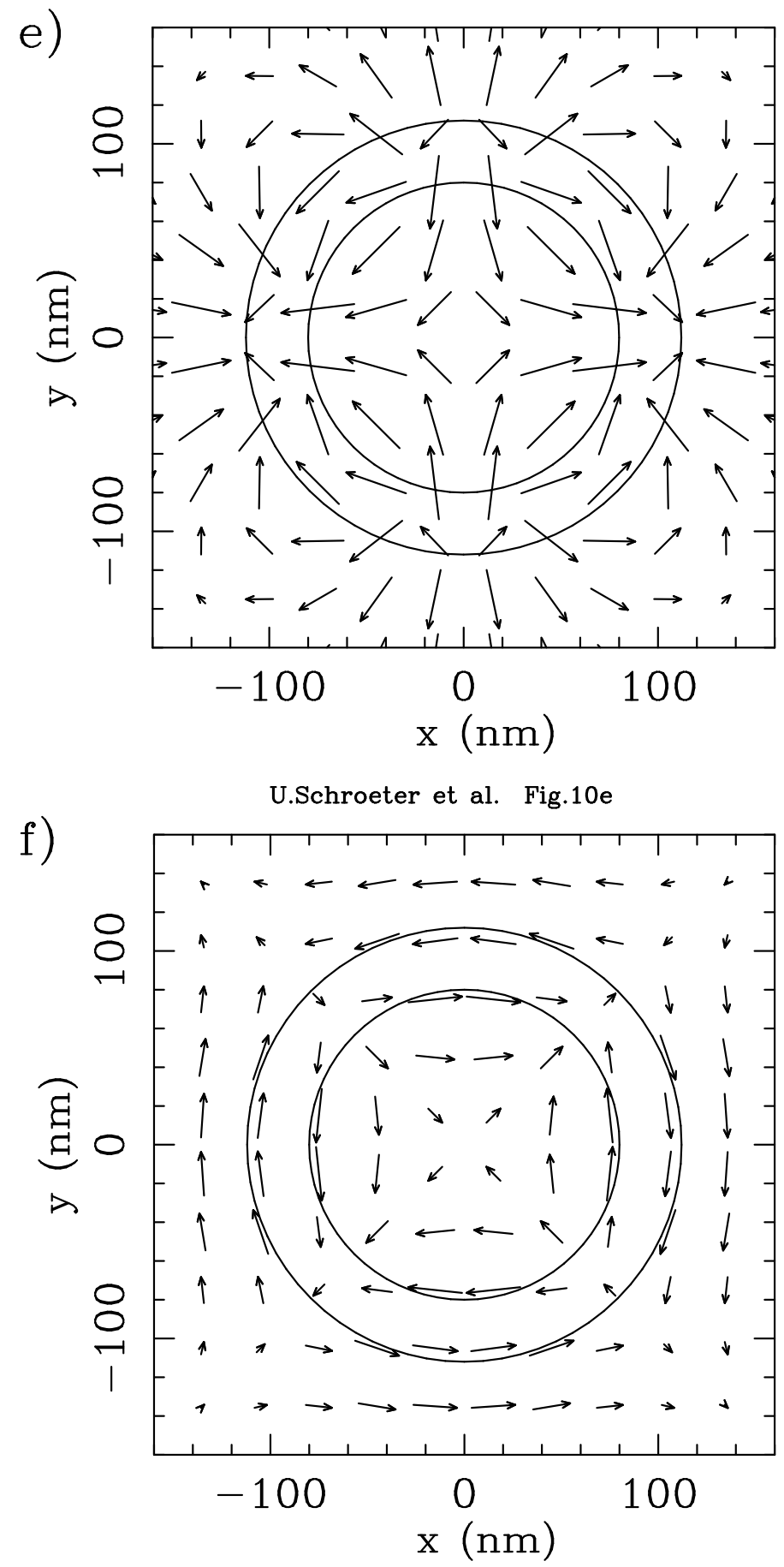

U.Schroeter et al. Fig.10f

FIG. 10. Arrow diagrams of electric and magnetic field in a plane perpendicular to the cylinder axis at a fixed time. The circles mark the core and outside boundaries of the cylinder. Arrows are placed with their center on the coordinate point they belong to and their lengths follow a logarithmic scale to avoid messy overlapping. The radial part of the electric field vector has three sign changes when going from the center to the outside of the cylinder, which might be not be easily seen due to the coarse arrow density in the diagrams. (a) Electric and (b) magnetic field for $n=0$, $\hbar c k_{z}=4.5 \mathrm{eV}, \hbar \omega=2.38 \mathrm{eV}$. (c) Electric and (d) magnetic field for $n=1, \hbar c k_{z}=4.5 \mathrm{eV}, \hbar \omega=2.37 \mathrm{eV}$. (e) Electric and (f) magnetic field for $n=2, \hbar c k_{z}=3.4 \mathrm{eV}, \hbar \omega=2.38 \mathrm{eV}$. 
a) $\mathrm{n}=0$

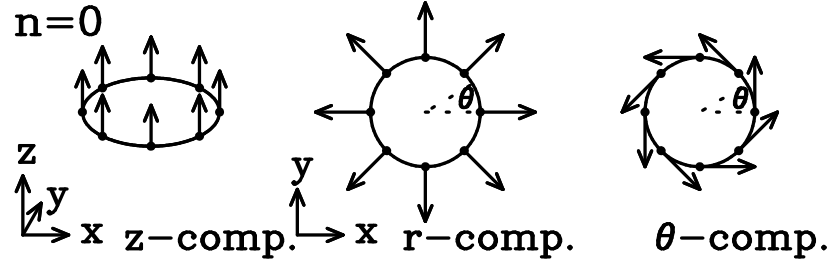

b) $n=1$
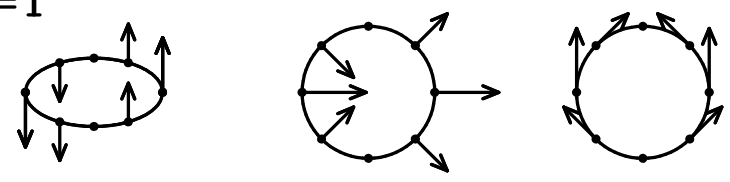

z-comp. $\quad$ r-comp. $\theta$-comp.

c) $\mathrm{n}=2$
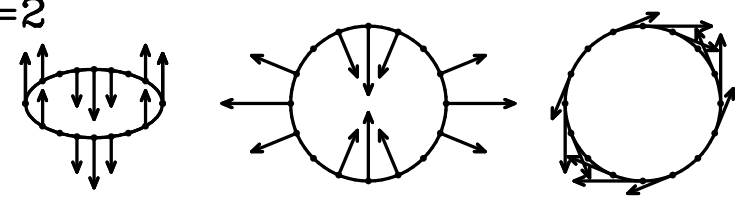

z-comp.

$\mathrm{r}$-comp.

$\theta$-comp.

d) $n=1$

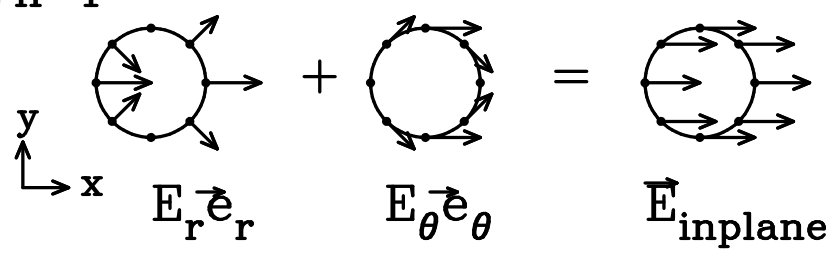

U.Schroeter et al. Fig.11

FIG. 11. (a) Field components are drawn separately for fixed radius $r$, fixed height $z$ and fixed time for (a) $n=0$, (b) $n=1$ and (c) $n=2$. (d) Radial and angular component of the electric field of the $n=1$ mode with their correct respective phase and resulting inplane field; the magnetic field components have to be added in the same way. (Differently to Fig.10, in this figure arrows are drawn as having their origin at the coordinates they belong to.) 


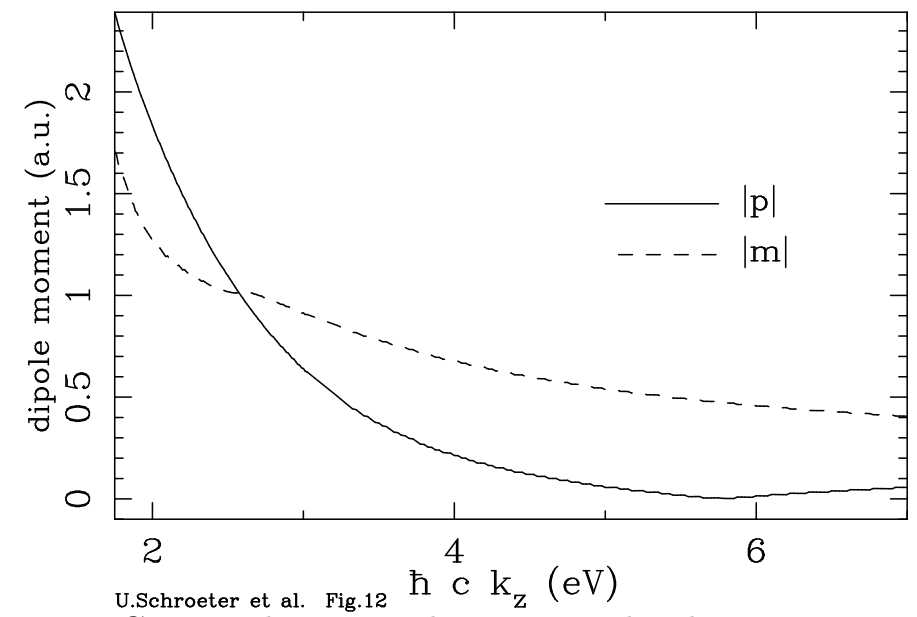

FIG. 12. Electric and magnetic dipole moment per unit length of the cylinder computed from the polarization currents along the low energy $n=1$ plasmon mode from Fig.2a, that is for cylinder radii $a=112 \mathrm{~nm}$ and $b=80 \mathrm{~nm}$. 